\title{
ELECTRIC FIELD EFFECTS ON GASIFICATION/COMBUSTION AT THERMO- CHEMICAL CONVERSION OF BIOMASS MIXTURES
}

\author{
Inesa Barmina $^{1}$, Maija Zake ${ }^{1}$, Harijs Kalis ${ }^{2}$, Maksims Marinaki ${ }^{2}$ \\ ${ }^{1}$ Institute of Physics, University of Latvia; \\ ${ }^{2}$ Institute of Mathematics and Computer Science, University of Latvia \\ mzfi@sal.lv, harijs.kalis@lu.lv
}

\begin{abstract}
A series of experimental studies and mathematical simulations of the electric field influence on the thermal decomposition of mixtures of wheat straw with wood and peat pellets and on the development of volatile combustion downstream the swirling flame flow was carried out. The main aim of these studies is to provide more efficient use of straw for cleaner and more efficient energy production by improving the gasification/combustion characteristics of the mixture and the composition of emission. The electric field influence on the combustion dynamics was studied experimentally using a pilot device, which combines a biomass gasifier and a combustor. An electric field was applied to the flame base using an axially inserted electrode. The electric field effect on the main gasification/combustion characteristics of a biomass mixture was estimated through complex measurements of the field-induced variations of the flow velocity, flame temperature, composition and heat output by varying the positive bias voltage of the axially inserted electrode. The mathematical model of the combustion process considers the electric field influence on the combustion characteristics using the approach of single chemical reaction. Simulations were performed for the opposite field configuration, when the negative bias voltage was applied to the axially inserted electrode by varying its length.
\end{abstract}

Keywords: biomass mixture, wood, peat, wheat straw, gasification, combustion dynamics, electric field.

\section{Introduction}

The use of the electric field (EF) to control the combustion characteristics for different types of hydrocarbon fuels (gaseous, liquid, solid) is considered as an effective tool to provide the local variations of flow dynamics, flame shape, structure, composition, temperature, heat output and composition of emissions, as well to improve the flame [1-3]. The results of the experimental study and theoretical analysis suggest that the main mechanism, which leads to the variation of the combustion dynamics can be related to the ion wind effect, when the electric field-induced body force initiates the acceleration of the flame ions $\left(\mathrm{CHO}^{+}, \mathrm{H}_{3} \mathrm{O}^{+}, \mathrm{C}_{2} \mathrm{H}_{3} \mathrm{O}^{+}, \mathrm{O}_{2}^{-}, \mathrm{OH}^{-}, \mathrm{CHO}_{3}^{-}\right)$in the field direction with subsequent momentum transfer to neutral flame species [1]. A detailed analysis of the processes developing in the industrial heat production devices when the electric field is applied to the flame reaction zone [4] demonstrates the usefulness of electrodynamic process control. In particular, the electric field allows controlling mixing of the flame species and provides homogenization of the flame reaction zone by improving the combustion conditions and by enhancing the heat transfer to the boiler heat surfaces. Similar improvement of the combustion characteristics was observed when the EF was used to control the main characteristics of district heating boilers [5]. The field-enhanced mixing of the flame components and the field-enhanced processes of heat/mass transfer to the boiler heat surfaces resulted in an increase of the heat energy production with a correlating increase of the boiler efficiency and in a decrease of the fuel consumption per unit of the produced heat energy and of the content of polluting $\mathrm{NO}_{\mathrm{x}}$ emission in the products. Moreover, the electric field application to control the processes developing at biomass thermo-chemical conversion has shown $[4 ; 5]$ that the electric field induced processes of heat and mass transfer can be used to control biomass thermal decomposition with improvement of the combustion characteristics. Assuming that the electric field can be applied to improve the biomass gasification/combustion characteristics, the present study is focused on more detailed experimental investigation and mathematical modelling of the electric field influence on the gasification/combustion characteristics of biomass mixtures (wheat straw, wood and peat pellets) with the aim to provide control of their thermo-chemical conversion and to ensure a more efficient use of the electric field control for cleaner heat energy production.

\section{Experimental studies}

The action of a DC electric field on the processes developing at thermo-chemical conversion of a wheat straw mixture with wood and peat pellets was experimentally studied using a batch-size pilotscale setup, which includes a biomass gasifier and a water-cooled combustor [5] with the inner 
diameter $60 \mathrm{~mm}$ and $650 \mathrm{~mm}$ of the total height. The gasifier is separated from the combustor by the inlet conical nozzle of the inner diameter $40 \mathrm{~mm}$. The initial weight of the biomass mixture, which was supplied to the gasifier, was $240 \mathrm{~g}$.

The primary air at the average rate $201 \cdot \mathrm{min}^{-1}$ was supplied to sustain the thermal decomposition of the biomass mixture. The secondary swirling air supplied at the average rate $30 \mathrm{l} / \mathrm{min}$ sustained the combustion of volatiles downstream the weakly swirled $(S=0.3-0.4)$ flame flow. The propane flame flow initiated the endothermic processes of biomass thermal decomposition, ignition and combustion of volatiles. The diagnostic sections with openings for the diagnostic tools (Pitot tube, thermocouples and gas sampling probes) provided the local measurements of flow velocity, flame temperature and composition. The local temperature of the flame reaction zone was measured by thermocouples (Pt$\mathrm{Pt} / \mathrm{Rh}$ ) and monitored using a Pico logger. The flame velocity profiles were measured with the help of a Pitot tube and a Testo 435 flowmeter. The local concentration of $\mathrm{O}_{2}, \mathrm{CO}_{2}, \mathrm{H}_{2}, \mathrm{CO}, \mathrm{NO}_{\mathrm{x}}$ and the combustion efficiency were measured by a gas sampling probe and by an analyzer Testo 350 . Calorimetric measurements of the cooling water flow were made for the estimation of the DC electric field effect on the heat output of the device. The water flow temperature was measured by thermo sensors AD 590 with online data registration by the Data Translation DT9805 acquisition module and Quick DAQ program. To estimate the electric field influence on the combustion dynamics at thermochemical conversion of biomass mixtures, a nichrome electrode, $3 \mathrm{~mm}$ in diameter and $35 \mathrm{~mm}$ in length, was placed at the bottom of the flame reaction zone and was positive with respect to the grounded walls of the inlet nozzle and to the water-cooled walls of the combustor. Therefore, the axially inserted electrode acts as the anode, whereas the inlet nozzle of the combustor and the grounded water-cooled walls of the combustor act as the cathode by collecting the ion current. To prevent a discharge, the current between the axially inserted electrode and the grounded walls of the combustor was limited to 5-7 mA at a voltage supply of $0.6-2.4 \mathrm{kV}$. The electrode voltage and the current were monitored by high voltage equipment.

\section{Results and discussion}

The experimental investigations of the electric field influence on the swirling flame flow behaviour and on the main combustion characteristics were conducted at thermo-chemical conversion of a mixture of wheat straw (20\%) with wood $(80 \%)$ and a mixture of wheat straw $(20 \%)$ with peat pellets $(80 \%)$ by comparing the main combustion characteristics of the undisturbed flame flow $(U=0)$ and of the flame, which was exposed to the electric body force $\left(F=n_{i} E\right)$. As follows from Fig. 1 , a-e, the electric body force disturbs the free flame shape and length. For the positive bias voltage of the axially inserted electrode, the electric body force leads to a decrease of the free flame length by increasing the visible radius of the flame reaction zone (Fig. 1, b, d). The reverse EF effect is observed for the negative bias voltage, when the flame is swirling around the electrode and stretches downstream the flame axis (Fig. 1, e).
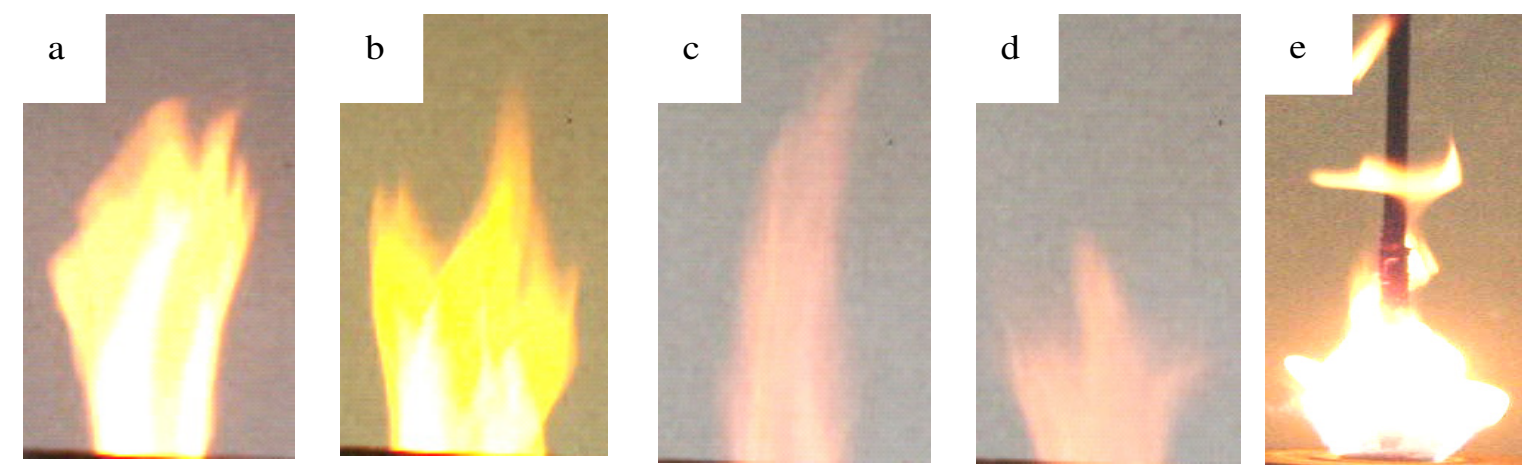

Fig. 1. Electric field effect on the free flame shape:

$$
\text { a, c }-U=0 ; \mathrm{b}, \mathrm{d}-U=+1.8 \mathrm{kV} ; \mathrm{e}-U=-1.2 \mathrm{kV}
$$

The results of previous research with the single electrode configuration [6] evidence of the fieldenhanced thermal decomposition of biomass (wood pellets) with the field-enhanced production of the axial flow of the main gasification products $\mathrm{CO}, \mathrm{H}_{2}, \mathrm{CH}_{4}$, unsaturated hydrocarbons $\left(\mathrm{C}_{2} \mathrm{H}_{2}, \mathrm{C}_{2} \mathrm{H}_{4}\right.$, etc. $)$ and primary ionic flame species. Among them, $\mathrm{CHO}^{+}, \mathrm{H}_{3} \mathrm{O}^{+}, \mathrm{C}_{2} \mathrm{H}_{3} \mathrm{O}^{+}, \mathrm{C}_{2} \mathrm{H}_{2}{ }^{+}, \mathrm{C}_{3} \mathrm{H}_{3}{ }^{+}$are the most likely 
ionic flame species [1]. The results of the present research confirm that increasing the positive bias voltage of the axially inserted electrode at the flame base results in the enhanced thermal decomposition of biomass mixtures accompanied by the field-enhanced formation of volatiles $\left(\mathrm{H}_{2}\right.$, $\mathrm{CO}$ ) during the primary pre-combustion stage (Fig. 2- a). The field-enhanced thermal decomposition of biomass mixtures is followed by the enhanced ignition and flaming combustion of the volatiles determining the formation of the main products $\mathrm{H}_{2} \mathrm{O}$ and $\mathrm{CO}_{2}$ with the correlating increase of the ion current towards the negatively biased inlet nozzle and water-cooled channel walls (Fig. 2- b, c). The maximum value of the ion current correlates with the formation of maximum values of the volume fraction of $\mathrm{CO}_{2}$ in the products and with the heat output from the flame reaction zone, and it rapidly decreases during the after-flame combustion stage (Fig. 2- b, c, d). Such correlation confirms that the dominant formation of flame ions occurs at the pre-combustion stage of biomass thermal decomposition and flaming combustion of volatiles, when the dominant electric field action on the combustion characteristics is observed, promoting the correlating increase of the flame temperature and combustion efficiency, whereas the mass fraction of the volatiles in the products decreases to the minimum value. As follows from Figures 2-b, c, d, similar field-induced variations of the combustion characteristics are observed for both mixtures of wheat straw with wood and with peat pellets.
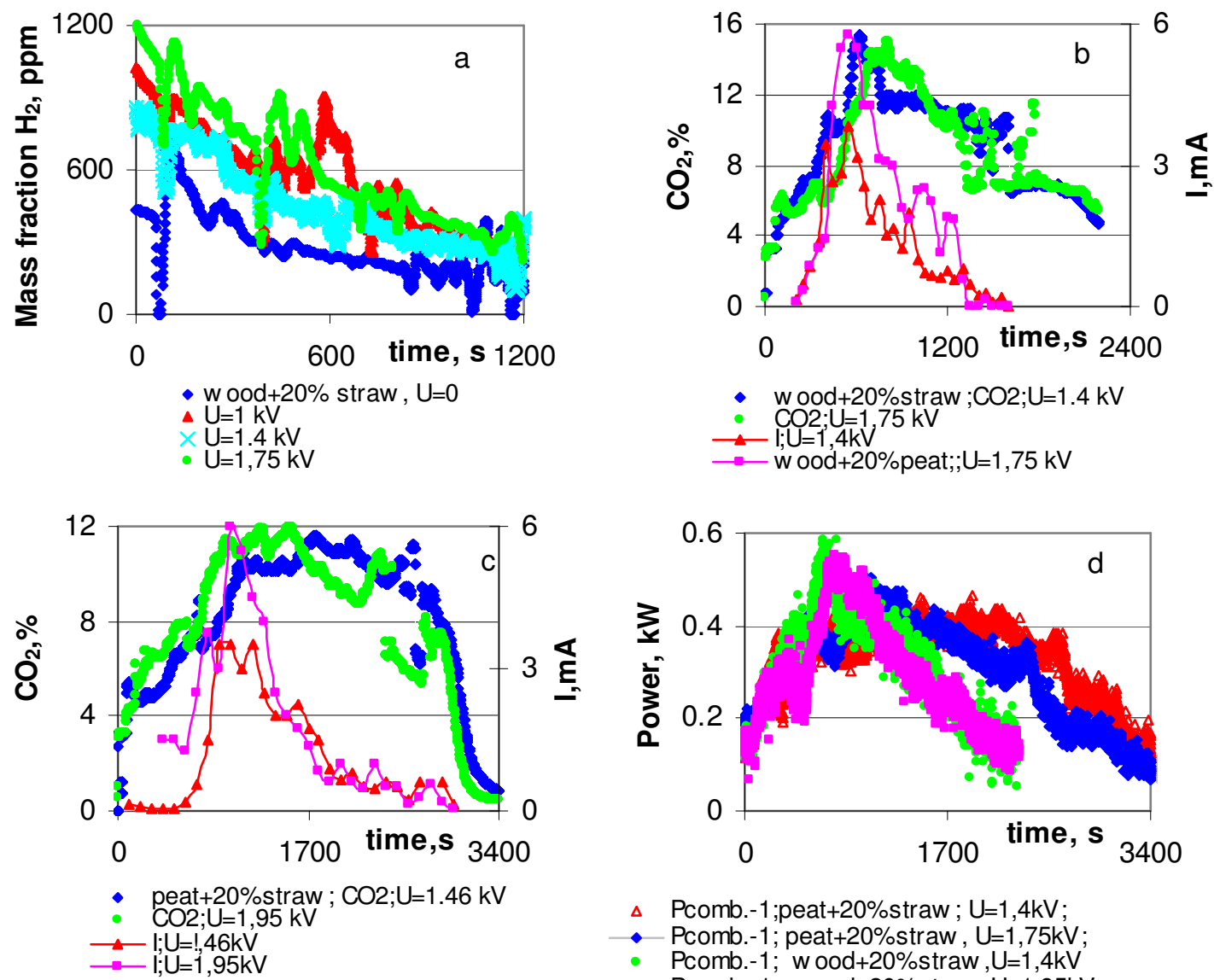

$\Delta \quad$ Pcomb.-1; peat+20\%straw; U=1,4kV;

- Pcomb.-1; peat+20\%straw, $U=1,75 \mathrm{kV}$;

- Pcomb.-1; w ood+20\%straw, $\mathrm{U}=1,4 \mathrm{kV}$

- Pcomb.-1; w ood+20\%straw ,U=1,95kV

Fig. 2. Electric field-induced time-dependent variations of the flame composition, ion current (a-c) and heat output from the device (d) at thermo-chemical conversion of wheat straw mixtures with wood and with peat pellets

The voltage dependence of the peak and average values of ion current demonstrates that with the given electrode configuration the increase of the positive bias voltage of the axially inserted electrode leads to the transition from the ion current saturation to a regime of space-charge controlled sheath convection of ions [7], with radial acceleration of ions toward the surface of the grounded inlet nozzle and with the correlating increase of the ion current (Fig. 3- a). The momentum transfer from ions to neutral flame species during their inelastic collisions promotes the radial mass transfer of neutrals completing the combustion of volatiles along the outside part of the flame reaction zone, which is highly responsible for the field-induced variations of the flame shape and length (Fig. 1) as well for 
the enhanced burnout of the volatiles (Fig. 2-b, c). However, by increasing the positive bias voltage of the axially inserted electrode and the ion current to the grounded channel walls, the radial acceleration of the charged flame species with the field-enhanced combustion of volatiles makes obvious its influence on the development of the flow dynamics. The results of the experimental study confirm the EF-induced variations of the flow dynamics. The applied EF promotes an increase of the axial flow velocity with correlating decrease of the average values of the tangential flow velocity and swirl intensity at the inlet of the combustor (Fig. 3-b). In addition, the local decrease of the peak values of the tangential flow velocity and flow swirl number along the outside part of the reaction zone is observed $(r / R>0.66)$. As a result of the competitive processes of field-enhanced combustion of volatiles and field-induced decrease of swirl intensity at the inlet of the combustor, the $\mathrm{CO}_{2}$ volume fraction (Fig. 3-c) in the products and the heat output from the reaction zone (Fig. 3-d) demonstrate the formation of maximum values at $I \approx 1 \mathrm{~mA}$ and start to decrease at $I>1 \mathrm{~mA}$, when the decrease of swirl intensity results in limited mixing of the axial flow of volatiles with the air swirl, with a correlating increase of the mass fraction of unburned volatiles in the products observed at thermochemical conversion of the biomass mixtures.
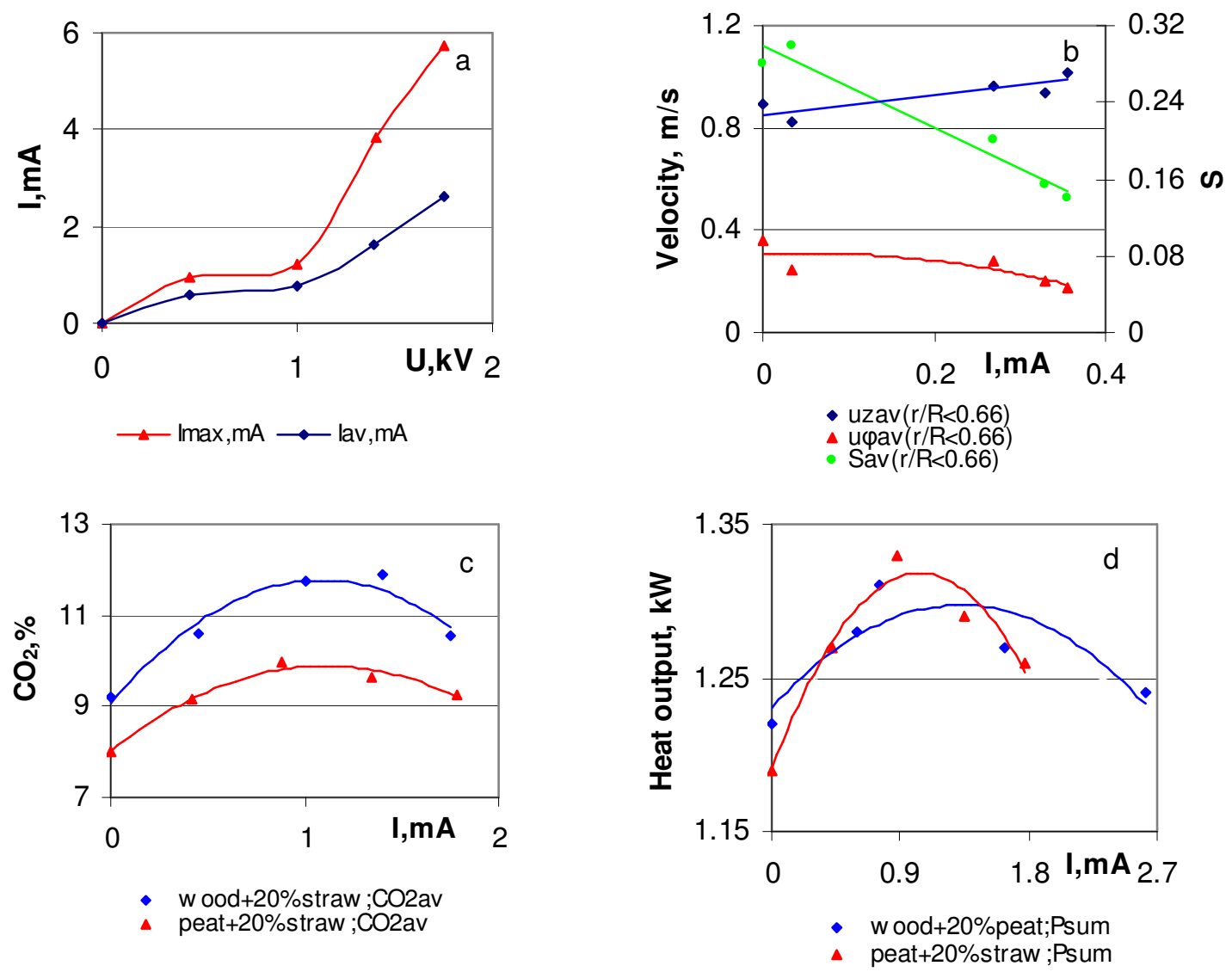

Fig. 3. Voltage dependence of the ion current (a); the flow velocity components (b), composition of the products (c) and heat output from the device (d) versus the ion current between the electrodes at thermo-chemical conversion of wheat straw mixtures with wood and with peat pellets

The results of previous research show [8] that the field-induced variations of the flame shape and length as well of the main characteristics of the flame reaction zone are affected by the polarity of the axially inserted electrode. With the negative bias voltage of the axially inserted electrode, the flame length increases, the flame is fastened on the electrode and stretches downstream the flame axis. This is confirmed by the results of the mathematical modelling of the field influence on the main flame characteristics for the negative bias voltage of the axially inserted electrode. 


\section{Mathematical modelling}

A numerical simulation of a 2D axially-symmetric, compressible, swirling flow (velocities $u_{r}, u_{z}$, $u_{\varphi}$ ), density $\rho$ ' was carried out at the development of the fuel burnout in a cylindrical pipe - combustor - with the radius $r_{0}=0.05 \mathrm{~m}$, length $z=z_{0}=0.1 \mathrm{~m}$. The primary axial air and the oxidant were supplied at $r^{\prime} \in\left[0, r_{1}^{\prime}\right]$ with the bulk velocity $U_{0}=0.01 \mathrm{~m} \cdot \mathrm{s}^{-1}$, density $\rho_{0}=1 \mathrm{~kg} \cdot \mathrm{m}^{-3}$; the inlet temperature was $T_{0}=300 \mathrm{~K}$ and the mass-fraction of fuel $C_{0}=1$. The secondary swirling air was supplied at $r^{\prime} \in\left[r_{1}{ }_{1}, r_{0}\right]$ with the bulk velocity $V_{0}>U_{0}$ and the swirl number $\left.S=V_{0} / U_{0}=3\right)$. The development of a simple chemical reaction (mass-fraction of the reactant $C^{\circ}$, temperature $T^{\circ}$ ) is described by four Euler, two reaction-diffusion and azimuthally induced magnetic field (component $\left.\mathrm{B}_{\varphi}\right)$ dimensionless equations in the cylindrical coordinates $(r, z)$ and at a time $\mathrm{t}$ [9]. The applied electric field induces an electrical current $\mathrm{I}$ of uniform density $\mathrm{j}_{0}$ between the positively biased walls of the combustor of length $L_{+}=\left\{r^{\prime}=r_{0}, 0<z<z_{2}\right\}$ and the negatively biased axially inserted electrode of length $L_{-}=\left\{r^{\prime}=r_{0} / 40,0<z<z_{1}\right\}$ determining the formation of the azimuthally induced magnetic field, which was scaled to $B_{0}=0.410^{-5} I \mathrm{~N} \cdot(\mathrm{A} \mathrm{m})^{-1}$. The influence of the variations in length of the axially inserted electrode and variations of the Lewis number on the flame characteristics is considered.

The equations were scaled using $r=r^{\prime} / r_{0}, x=z / r_{0}, x_{0}=z_{0} / r_{0}=2, x_{1}=z_{1} / r_{0}, x_{2}=z_{2} / r_{0}, r_{1}=r^{\prime}{ }_{1} / r_{0}=$ 0.75), $\rho=\rho^{\prime} / \rho_{0}, u=u_{r} / U_{0}, w=u_{z} / U_{0}, T=T^{\prime} / T_{0}, C=C^{\prime} / C_{0}, B_{f}=B_{\varphi} / B_{0}$ and the circulation $v=u_{\varphi} r$ to $V_{0} r_{0}$. For the dimensionless pressure $\mathrm{p}$, we consider a model for perfect gas: $p=\rho \cdot T$. The dimensionless radial and axial components of electromagnetic forces were quantified with the help of the parameter $P_{e}=B_{0} j_{0} r_{0} /\left(\rho_{0} U_{0}^{2}\right)$.

The exothermic chemical reaction is modelled using a single step reaction between the fuel and the oxidant $\left(\mathrm{O}_{2}\right)$ producing heat and products as follows:

$$
\left\{\begin{array}{l}
\frac{\partial T}{\partial t}+u \frac{\partial T}{\partial r}+w \frac{\partial T}{\partial x}=\frac{L e}{\rho P e} \Delta T+\beta A C \exp \left(-\frac{\delta}{T}\right) \\
\frac{\partial C}{\partial t}+u \frac{\partial C}{\partial r}+w \frac{\partial C}{\partial x}=\frac{1}{\rho P e} \Delta C-A C \exp \left(-\frac{\delta}{T}\right)
\end{array}\right.
$$

where $P e=\frac{\rho_{0} U_{0} r_{0}}{D}, L e=\frac{\lambda}{c_{p} D}$ are the Peclet and Lewis numbers

$\left(P_{1}=L e / P e, P_{2}=1 / P e, L e=P_{1} / P_{2}\right)$,

$\beta=\frac{B}{c_{p} T_{0}}=5$ is the heat-release parameter,

$\delta=\frac{E}{R T_{0}}=10$ is the scaled activation-energy,

$A=A^{\prime} r_{0} / U_{0}=50000$ is the scaled pre-exponential factor.

Here, the molecular diffusivity $D=5 \cdot 10^{-5} \mathrm{~m}^{2} \cdot \mathrm{s}^{-1}$, the thermal conductivity $\lambda=5 \cdot 10^{-2} \mathrm{~J} \cdot(\mathrm{s} \cdot \mathrm{m} \cdot \mathrm{K})^{-1}$, the specific heat capacity $c_{p}=10^{3} \mathrm{~J} \cdot(\mathrm{kg} \cdot \mathrm{K})^{-1}$, the specific heat release $B=1.5 \cdot 10^{6} \mathrm{~J} \cdot \mathrm{kg}^{-1}$, the reactionrate pre-exponential factor $\mathrm{A}^{\prime}=10^{4} 1 \cdot \mathrm{s}^{-1}$, the activation energy $E=2.5 \cdot 10^{4} \mathrm{~J} \cdot \mathrm{mol}^{-1}$, R is the universal gas constant, $P_{1}=P_{2}=0.1, L e=1$. The decrease of the molecular diffusivity to $D=5 \cdot 10^{-6} \mathrm{~m}^{2} \cdot \mathrm{s}^{-1}$ and thermal conductivity to $\lambda=5 \cdot 10^{-3} \mathrm{~J} \cdot(\mathrm{s} \cdot \mathrm{m} \cdot \mathrm{K})^{-1}$ results in the corresponding decrease of $P_{2}$ to $P_{2}=0.01$ and $P_{1}$ to $P_{1}=0.01$. For $\beta=0.5, B^{\prime}=1.5 \cdot 10^{5} \mathrm{~J} \cdot \mathrm{kg}^{-1}$.

The hydrodynamic stream function was approximated in the following way: $r \rho w=\partial \Psi / \partial r$, $r \rho u=-\partial \Psi / \partial x$.

At the inlet of the combustor $x=0$, the following boundary conditions (BC) were applied: $u=0, T=1$ for $r \in[0,1]$, and $w=1, C=1, \Psi=0.5 r^{2}, v=0$ for $r \in\left[0, r_{1}\right] ; \Psi=q, v=4 r \frac{\left(r-r_{1}\right)(1-r)}{\left(1-r_{1}\right)^{2}}$, $w=0, C=0$ at $r>r_{1},\left(q=r_{1}^{2} / 2=0.28125\right.$ is the dimensionless fluid volume $), r_{1}=0.75$. The uniform jet flow was considered at $r<r_{1}$ and the air swirl motion was considered at $r>r_{1}$ with the maximum 
azimuthal velocity equal to unity. For the magnetic induction $B_{f}$ at $L_{+}: B_{f}=1-x / x_{2}$ for $x<\left[0, x_{2}\right]$ and $B_{f}=0$ for $x>x_{2}$; at $L_{:}: B_{f}=\left(1-x / x_{1}\right) / 40$ for $x \in\left[0, x_{1}\right]$ and $B_{f}=0$ for $x>x_{1}$; at $x=0: B_{f}=1 / r$.

For the numerical calculation, the implicit finite difference scheme and the ADI method were applied providing the estimation of the effects of the variation of the molecular diffusivity, thermal conductivity and electric field on the main flame characteristics, i.e. on the minimum value of flow density, on maximum values of the flow velocity components, on the reaction rate $R^{*}=A C \exp (-\delta / T)$, on the pressure gradient and on the stream function. The influence of the molecular diffusivity and thermal conductivity on the main characteristics of the undisturbed flame flow for $\beta=5$ and $P_{e}=0$ is summarized in Table 1 . These results show that with the constant molecular diffusivity D (Table 1, no. $1,2)$ a decrease in thermal conductivity $\lambda\left(P_{1}=0.01, L e=0.1\right)$ leads to an increase in maximum values of the flow velocity components $(w)$, temperature, reaction rates, flow vortices with a decrease in the flow density, whereas with the constant thermal conductivity $\lambda$ (Table 1 , no. 1,3 ) the decrease in molecular diffusivity $D\left(P_{2}=0.01, L e=10\right)$ results in an increase in maximum density and in a decrease of the velocity, pressure gradient, temperature, reaction rate and flow vortices.

Table 1

Effect of molecular diffusivity and thermal conductivity on the main flame characteristics for $\beta=5, P_{e}=0$.

\begin{tabular}{|c|c|c|c|c|c|c|c|c|c|c|}
\hline No & \multirow{2}{*}{$\boldsymbol{P}_{\mathbf{1}}$} & $\boldsymbol{P}_{\mathbf{2}}$ & $\boldsymbol{m i n}$ & $\boldsymbol{m a x}$ & $\boldsymbol{m a x}$ & $\boldsymbol{m a x}$ & $\boldsymbol{\Delta}$ & $\boldsymbol{m a x}$ & \multirow{2}{*}{$\boldsymbol{\boldsymbol { T } _ { \boldsymbol { v } }}$} & $\begin{array}{c}\max \\
\boldsymbol{\Psi}\end{array}$ \\
\hline 1 & 0.01 & 0.01 & .0286 & 5.817 & 3.203 & 6.0000 & 4.27 & 98.71 & 5.824 & 0.2943 \\
\hline 2 & 0.10 & 0.01 & .0425 & $\mathbf{3 . 7 0 1}$ & 1.767 & $\mathbf{2 . 4 6 7}$ & 2.04 & 61.22 & $\mathbf{2 . 2 0 7}$ & 0.2854 \\
\hline 3 & 0.01 & 0.10 & .0228 & $\mathbf{9 . 4 3 3}$ & 5.254 & $\mathbf{1 5 . 8 1 1}$ & 9.78 & 401.2 & 15.27 & $\mathbf{0 . 3 5 5 7}$ \\
\hline 4 & 0.10 & 0.10 & .0305 & 5.798 & 2.785 & 5.9940 & 4.25 & 298.8 & 5.193 & 0.2924 \\
\hline
\end{tabular}

A similar effect of the variation of molecular diffusivity and thermal conductivity on the main flame characteristics was found when the electric field was imposed on the flame reaction zone $(\beta=5$; $\left.P_{e}=0.05\right)$. For the fixed length of the axially inserted electrode $\left(x_{1}\right)$, the decrease in conductivity $\lambda$ $\left(P_{1}=0.01, L e=0.1\right)$ resulted in the correlating increase of the maximum velocity, pressure gradient, flame temperature, reaction rate and flow vortices $(\max \Psi>0.28125)$ along with the decrease in density, whereas the decrease of the molecular diffusivity $D\left(P_{2}=0.01, L e=10\right)$ resulted in the increase of the maximum density and in the decrease of the velocity, pressure gradient, temperature, reaction rate and flow vortices (Table 2). With the fixed values of $D$ and $\lambda\left(P_{1}=\mathrm{P}_{2}=0.1, L e=1\right)$, the electric field affects the flow dynamics, the local and average values of the flow velocity, the density and pressure in the flame reaction zone. The electric field influence on the main flame characteristics is summarized in Table 2.

Table 2

Electric field effect on the main flame characteristics for different length $x_{1}$ of the axially inserted electrode, $P_{1}$ and $P_{2}$ for $\beta=5, P_{e}=0.05, x_{2}=2$

\begin{tabular}{|c|c|c|c|c|c|c|c|c|c|c|c|}
\hline $\begin{array}{c}x_{1} \\
\text { length }\end{array}$ & $P_{1}$ & $P_{2}$ & $\underset{\rho}{\min }$ & $\underset{w}{\max }$ & $\begin{array}{c}\max \\
u\end{array}$ & $\underset{u}{\min }$ & $\underset{T}{\max }$ & $\Delta p$ & $\begin{array}{c}\max \\
R\end{array}$ & $\boldsymbol{T}_{a v}$ & $\underset{\Psi}{\max }$ \\
\hline 0.25 & 0 & 1 & 0207 & 7.486 & 3.137 & 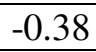 & 6.0000 & 20 & 122.7 & .821 & 0.2944 \\
\hline 0.25 & 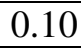 & & & & & & & 4.16 & & & \\
\hline 0.25 & 0.10 & 0.01 & & & & -0.47 & & 2.02 & & 2.227 & \\
\hline 0.25 & 0.01 & 0.10 & & & & -0.31 & & 9.66 & 401.3 & 15.11 & 3067 \\
\hline 0.5 & 0.01 & 0.01 & 0.02 & 7.9 & & -0.26 & & 4.21 & 10 & 5.821 & 2944 \\
\hline 1 & 0.0 & & & & & -0.16 & & 4.22 & 98.78 & 5.822 & .2944 \\
\hline 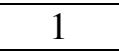 & & & & & & -0 & & 9.70 & 3 & 15.21 & 629 \\
\hline 1 & & & & & & -0.2 & & 1.98 & 03 & 2.215 & 854 \\
\hline 1 & .10 & .10 & 0.0318 & 9.027 & 2.724 & -0.15 & 5.9951 & 4.18 & 298.9 & 5.199 & 0.2924 \\
\hline
\end{tabular}

The results of the numerical simulation suggest that the electric field influence on the main flame characteristics is determined by the length of the axially inserted electrode, i.e. the increase in length of the electrode, which results in the correlating increase of the maximum value of the flow velocity and pressure gradient (Table 2). With the fixed length of the electrodes $\left(x_{1}=0.25\right)$ and fixed values of 
$P_{1}$ and $P_{2}\left(P_{1}=0.01, P_{2}=0.1\right)$, the application of the electric field to the flame reaction zone leads to an increase in length of the flame reaction zone (Fig. 4 a, b); the electric field disturbs the stream lines, initiates the formation of small vortices, and the radial velocity changes the sign (Fig. $5 \mathrm{a}, \mathrm{b}$ ). The levels of the electric current decrease when increasing the distance from the top of the electrode (Fig. 6 -a), and the flame temperature ( $t=0.2 \mathrm{~s}$ ) rapidly increases to its maximum value (Fig. $6 \mathrm{~b}$ ).

a

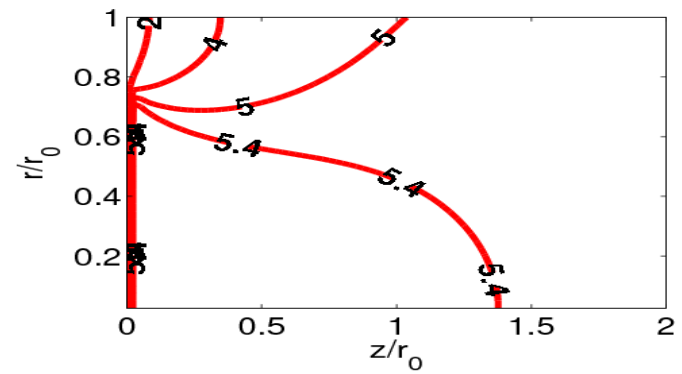

$\mathrm{b}$

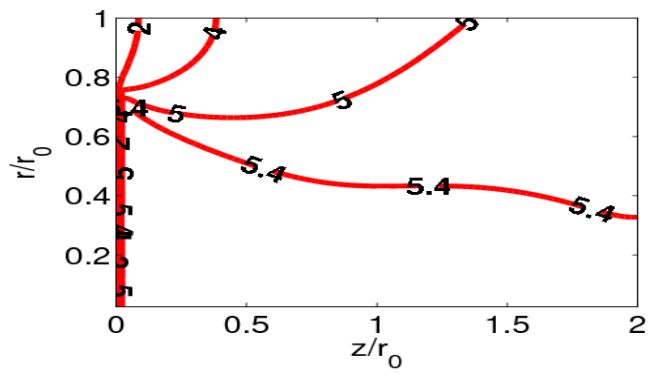

Fig. 4. Temperature levels for $\boldsymbol{P}_{1}=\mathbf{P}_{2}=\mathbf{0 . 1}, \boldsymbol{P}_{e}=\mathbf{0}, \max T=\mathbf{5 . 9 9 4 5}, \boldsymbol{T}_{a v}=\mathbf{5 . 1 9 2 6}$ (a) and for $P_{e}=0.5, \max T=5.9973, T_{a}=5.2113, x_{1}=0.25, x_{2}=2(\mathrm{~b})$

a

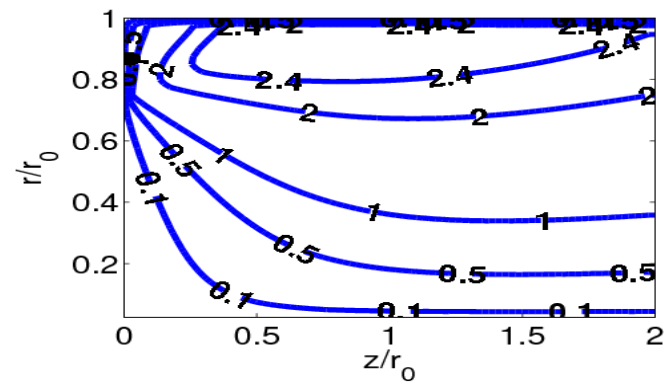

b

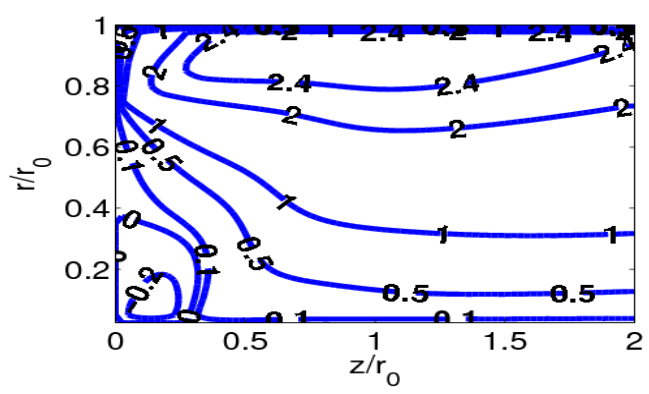

Fig. 5. Levels of the radial velocity for $P_{1}=P_{2}=0.1, P_{e}=0, \max u=2.786$ (a) and for $P_{e}=0.05, \max u=2.782, \min u=-0.36(\mathrm{~b})$

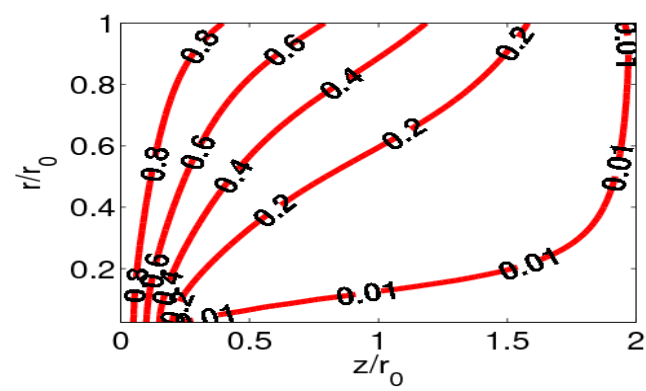

b

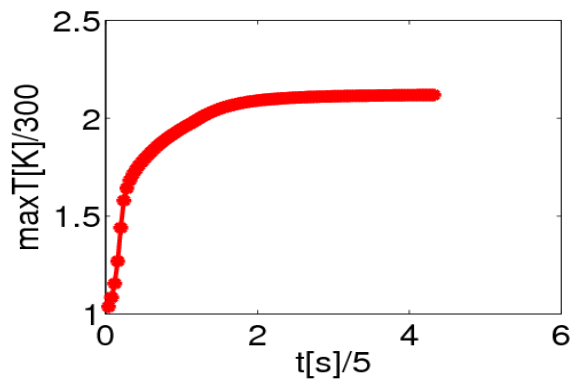

Fig. 6. Levels of the electric current for $x_{1}=0.25, x_{2}=2$ (a); maximum temperature dependence for $\beta=0.5, P_{1}=0.01, P_{2}=0.1(b)$

\section{Conclusions}

The experimental study, mathematical modelling and numerical simulation of the electric field influence on the combustion characteristics at thermo-chemical conversion of wheat straw mixtures with wood and peat pellets for the field configuration with the positively and negatively biased axially inserted electrode have led to the following conclusions.

1. With the positive bias voltage of the axially inserted electrode, the electric field body force leads to the enhanced thermal decomposition of the biomass mixtures, to the field-enhanced combustion of the volatiles, with the correlating increase of the ion current, combustion efficiency, $\mathrm{CO}_{2}$ volume fraction in the products and of the heat output from the device up to their peak values during the flaming combustion of volatiles, with rapid decrease of the field effect on the combustion characteristics at the after-flame smouldering stage.

2. The voltage dependence of the main flame characteristics suggests that the electric field-enhanced thermo-chemical conversion dominates at $U<1 \mathrm{kV}$ and starts to decrease at $U>1 \mathrm{kV}$, when the radial acceleration of the flame ions along with the field-induced radial mass transfer of the 
neutral flame species slows down the swirl intensity, which is highly responsible for the mixing of the flame components and for the formation of the flame reaction zone.

3. With the negatively biased axially inserted electrode, the electric field exhibits its influence on the flow dynamics, on the local, average and maximum values of the flow velocity, density and pressure in the flame reaction zone, advancing so the formation at the pipe inlet of small vortices with anti-clockwise rotation direction and increasing the flame length, and the radial velocity changes the sign.

4. The field influence on the flame characteristics depends on the length of the electrode, i.e. increasing the length of the axially inserted electrode leads to the increase in maximum velocity and in pressure gradient.

\section{Acknowledgements}

The authors would like to express their gratitude for the financial support from the Latvian research grant ZD2015/AZ83, European Regional Funding for project SAM 1.1.1.1./A/16/004 and Latvian Research Cooperation Project of the Latvian Concil of Science Nr. 623/2014.

\section{References}

1. Lawton J., Weinberg F. Electric Aspects of Combustion. Clarendon Press, Oxford, England, $1969,650 \mathrm{p}$.

2. Gan Y., Wang M., Luo Y, Chen X., Xu J., Effects of direct-current electric fields on flame shape and combustion characteristics of ethanol in small scale, Advances in Mechanical Engineering, 2016, vol. 8 (1), pp. 1-14.

3. Xie L., Kishi T., Kono M. The influences of electric fields on soot formation and flame structure of diffusion flames, Journal of Thermal Science, 1993, vol. 2, issue 4, pp. 288-293.

4. Colannino, J. Electrodynamic Combustion Control TM Technology. A Clear Sign White Paper, 2012, ClearSign Combustion Corporation, Seattle.

5. Barmina I., Purmalis M., Valdmanis R., Zake M. Electrodynamic control of the combustion characteristics and heat energy production. Combustion Science and Technology, 2016, vol. 188 (2), pp. 190-206.

6. Barmina I., Kolmičkovs A., Valdmanis R., Vostrikovs S., Zake M, 2015, Electric field control of biomass gasification. Proceedings International Conference "Engineering for Rural Development", 2015, Jelgava, pp. 510-515.

7. Smy P.R., The use of Langmuir probes in the study of high pressure plasmas. Advances in Physics, 1976, vol. 25, no. 5, pp. 517-553.

8. Zake M., Barmina I., Turlajs D., Ramata A., Meijere A. Experimental study of the electric fieldenhanced heat transfer from swirling combustion. Proceedings CHISA-2004, paper 0079, Prague, pp. 1-6.

9. Kalis H., Barmina I., Zake M., Koliskins A. Mathematical Modelling and Experimental Study of Electrodynamic Control of Swirling Flame Flows. Proceedings International Conference "Engineering for Rural Development", 2016, Jelgava, pp. 134-141. 